\title{
Xantomatosis eruptiva: presentación de un caso y revisión de la literatura
}

\author{
Giovani Andrés Rojas-Martelo ${ }^{1}$; Diana Katherine Hernández-Quiceno ${ }^{2}$; Walter Espinoza-Delgado ${ }^{3}$
}

${ }^{1}$ Medicina Familiar y Comunitaria, Centro de Salud Jazmín, Madrid, España.

${ }^{2}$ Unidad de Cuidado Crítico, Clínica La Estancia, Popayán, Colombia.

${ }^{3}$ Servicio de Dermatología, Hospital Universitario Ramón y Cajal, Madrid, España.

\section{Correspondencia:}

giovani_rojas@yahoo.com

Dirección: Avenida Manoteras, 38 portal A piso 3ำ puerta 307,

Madrid, España, CP: 28050. Teléfono: +34657641437

dn_andina@yahoo.com

espinoza_delgado_walter@hotmail.com

Fecha de recepción: 10/10/2017

Fecha de aceptación: 18/10/2017

\section{Resumen}

L os xantomas eruptivos son depósitos anormales de lípidos en la piel los cuales se asocian a células espumosas, representadas por macrófagos cargados de lípidos. Están relacionados con trastornos del metabolismo lipídico y, con menor frecuencia, a gammapatías monoclonales. En un porcentaje de los casos no se evidencia una enfermedad subyacente apareciendo estas lesiones de manera esporádica. Presentamos el caso de un varón de 57 años con antecedentes de diabetes mellitus y dislipidemia que presenta lesiones pápulonodulares amarillentas diseminadas en el cuerpo, compatibles clínica e histológicamente con xantomas eruptivos.

Palabras clave: xantomatosis, dislipidemia, diabetes mellitus.

\section{Abstract}

Eruptive xanthomas are abnormal deposits of lipids and foam cells (represented by lipid-laden macrophages) in the skin. They are related to disorders of lipid metabolism and, less frequently, to monoclonal gammopathies. In a percentage of the cases there is no evidence of a subsequent disease appearing these lesions sporadically. We report the case of a 57-year-old male with a prior history of diabetes mellitus and dyslipidemia, with yellowish papulo-nodular lesions disseminated in the body, clinically and histologically compatible with eruptive xanthomas.

Key words: xanthomatosis, dyslipidemia, diabetes mellitus.

\section{Introducción}

Los xantomas representan depósitos de lípidos en la piel. Clínicamente se clasifican en eruptivos, tuberoeruptivos o tuberosos y tendinosos o planos ${ }^{(1)}$. Los xantomas eruptivos se caracterizan por presentarse como lesiones papulosas de 1 a $4 \mathrm{~mm}$ de diámetro, de coloración amarillenta-anaranjada de base inflamatoria ${ }^{(2)}$ que suelen distribuirse principalmente en extremidades. Ocasionalmente son pruriginosas o incluso pueden producir dolor. En algunos casos se ha descrito fenómeno de Köebner ${ }^{(3)}$. Histológicamente presentan depósitos lipídicos en dermis reticular con infiltrado inflamatorio mixto conformado por neutrófilos y linfocitos. A diferencia de otros tipos de xantomas, los xantomas eruptivos presentan células espumosas pequeñas y aparecen en menor cantidad. Los lípidos extracelulares se hallan en la dermis como artefactos de preparación, como un material amorfo de color azul-grisáceo ${ }^{(4)}$.

Entre los principales diagnósticos diferenciales a considerar ante la presencia de este tipo de lesiones se encuentran: el xantoma diseminado, los xantomas tendinosos, los xantomas tuberosos, histiocitomas eruptivos, xantomas verruciformes, xantogranuloma juvenil, granuloma anular diseminado, molluscum contagiosum y xantogranuloma necrobiótico ${ }^{(2,5)}$.

\section{Caso clínico}

Presentamos el caso de un varón de 57 años con antecedentes de hipertensión arterial en tratamiento con olmesartán/amlodipino 40/10 mg al día, atenolol $50 \mathrm{mg}$ al día e hidroclorotiazida $50 \mathrm{mg}$ al día; dislipidemia en tratamiento con atorvastatina $10 \mathrm{mg}$ en las noches y fenofibrato $145 \mathrm{mg}$ al día; diabetes mellitus tipo 2 en tratamiento con metformina 850 mg, un comprimido cada 12 horas. Acude a consulta de atención primaria para seguimiento de sus enfermedades crónicas, realizándose analítica correspondiente. El paciente se encontraba asintomático, salvo por las lesiones que presentaba en piel, de seis meses de evolución a las cuales se refirió solo en el interrogatorio dirigido. Refiere poca adherencia al tratamiento. Su última visita a consulta de atención primaria fue hace dos años.

En la exploración física destaca un índice de masa corporal compatible con obesidad (IMC: $33 \mathrm{Kg} / \mathrm{m}^{2}$ ) y lesiones 
eruptivas papulonodulares múltiples de aproximadamente 2-4 mm, de color amarillento, compatibles con xantomas diseminados en todo el cuerpo, no dolorosas ni pruriginosas, de localización predominante en rodillas y antebrazos (figura 1). En la analítica destaca sodio $121 \mathrm{mmol} / \mathrm{l}$, cloro $86 \mathrm{mmol} / \mathrm{l}$, colesterol total $1383 \mathrm{mg} / \mathrm{dL}$, cHDL $22 \mathrm{mg} / \mathrm{dL}$, cLDL $443 \mathrm{mg} /$ $\mathrm{dL}$, triglicéridos $>5680 \mathrm{mg} / \mathrm{dL}$, glucosa $289 \mathrm{mg} / \mathrm{dL}$, HbA1c: 13,6\%, osmolalidad (medida en suero): $412 \mathrm{mmol} / \mathrm{kg}$. Resto de determinaciones sin hallazgos relevantes. Se realizó biopsia de una de las lesiones mediante punch de piel de muslo, cuyo informe de anatomía patológica es compatible con xantoma eruptivo (figura 2).

\section{Discusión}

Los xantomas eruptivos suelen estar asociados a trastornos metabólicos como la hipertrigliceridemia, por lo que es imprescindible una adecuada anamnesis en donde se incluyan los antecedentes patológicos personales y familiares. La realización de una analítica con perfil lipídico, glucemia, hemoglobina glicosilada, función renal y perfil hepático constituye un punto de partida fundamental para establecer el diagnóstico y evaluar posibles causas secundarias. A su vez, ante los hallazgos de lípidos y lipoproteínas considerablemente alterados con respecto a los valores de referencia y la evidencia de depósitos de lípidos en zonas atípicas es razonable plantear una etiología primaria de este trastorno metabólico ${ }^{(6)}$.

El tratamiento de estas lesiones se basa en un abordaje multidisciplinario del trastorno metabólico de base, pasando por el médico de atención primaria, quien deberá inculcar estilos de vida saludable, orientando una dieta equilibrada y baja en grasa, individualizada a las características y necesidades del paciente, fomentando el ejercicio, realizando seguimiento de las medidas instauradas para lograr un peso adecuado y prescribiendo la terapéutica farmacológica inicial, según el tipo de dislipidemia, el riesgo cardiovascular y las comorbilidades que presente el paciente. En el caso de dislipidemias, familiares o en las que no se logran los objetivos terapéuticos, una derivación oportuna a especialidades como endocrinología, dermatología, genética y medicina interna, ofrece la posibilidad de realizar un manejo óptimo del paciente ${ }^{(4-8)}$.

Con un adecuado control metabólico enfocado en reducir los niveles de colesterol de baja densidad (cLDL) y niveles de triglicéridos, no solo se logra reducir o incluso desaparecer los xantomas eruptivos, sino que también se reduce el riesgo de eventos cardiovasculares y pancreatitis, que son las entidades que suelen determinar el pronóstico de estos pacientes ${ }^{(4,9,10)}$.

En conclusión, presentamos el caso de un varón en la quinta década de la vida que presenta dislipidemia, mixta que, tras el abandono de su tratamiento, presenta lesiones diseminadas en piel, compatibles clínica e histológicamente con xantomas eruptivos. Al realizar analítica se objetivan niveles de triglicé-
Figura 1. Lesiones compatibles con xantomas eruptivos en rodillas y antebrazo

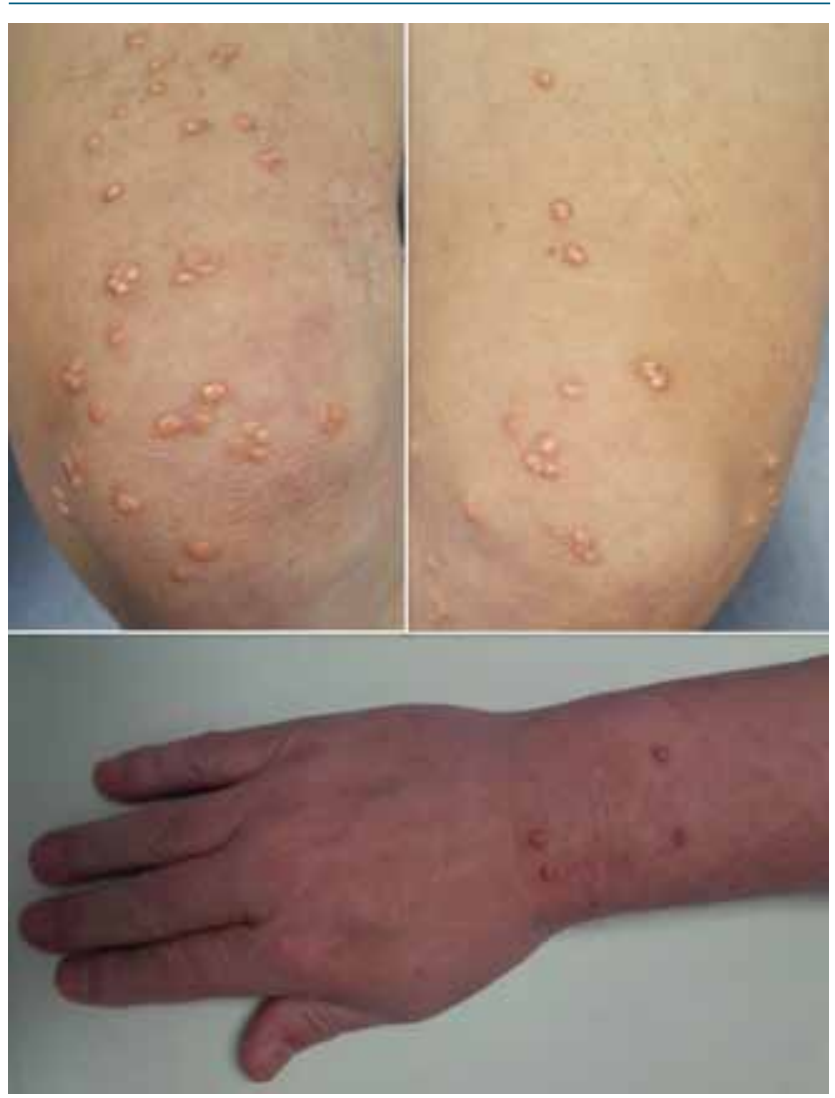

Figura 2. Estudio histológico de la lesión compatible con xantoma eruptivo (H\&E X10)

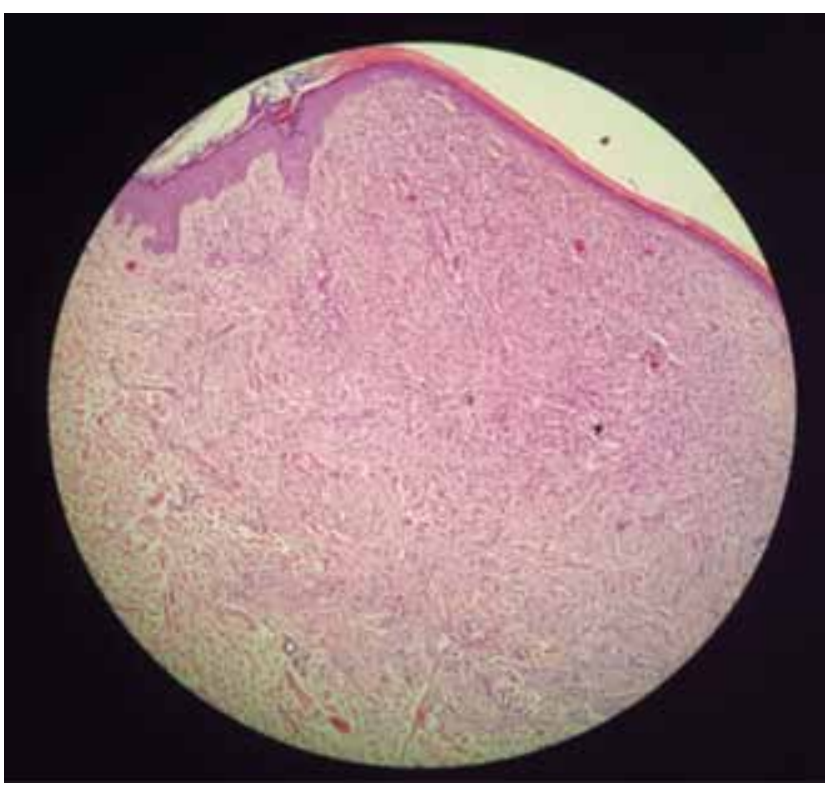


ridos y colesterol llamativamente elevados y escaso control glucémico evidenciado por los niveles de hemoglobina glucosilada. Tras reiniciar la terapéutica con una adherencia ade- cuada y con seguimiento conjunto con el servicio de endocrinología, se evidenció mejoría del perfil lipídico con reducción importante de los xantomas.

\section{Referencias}

1. Violini V, Meneses M, Samper A. Xantomatosis eruptiva asociada a hiperlipoproteinemia tipo IV de Fredrickson A propósito de un caso. 2014;64(6):230-3.

2. Villalón G, Martín JM, Monteagudo C, Alonso V, Montesinos E, Jordá E. Xantomas eruptivos en el debut de diabetes mellitus. Actas Dermosifiliogr. 2008;99(5):426-7.

3. Goldstein GD. The Koebner response with eruptive xanthomas. J Am Acad Dermatol, 1984:10(6):1064-5.

4. Gámez Pérez L, Reig Macías I, Gutiérrez Paredes E, Monteagudo Castro C, Jordá Cuevas E. Xantomas eruptivos. Causas, diagnóstico y tratamiento. Piel. 2011;26(10):505-7.

5. Sacchi A, Olivares L. Xantomatosis y dislipoproteinemias: Dermatol. Argent, 2013, 19(3): 174-182

6. Brites FD, Gómez Rosso LA, Boero LE, Rivera S. Clasificación y diagnóstico bioquímico de las dislipemias. Síndrome Metabólico y Riesgo Vascular. Buenos Aires; 2012;150-68

7. Laufs U, Parhofer KG. Simplified algorithm to facilitate communication of familial hypercholesterolaemia. Eur Heart J. 2015;36(43):3004-6.

8. Cepeda LL, Ramos-garibay JA, Calderón DP. Xantomas eruptivos como manifestación inicial de diabetes mellitus e hipertrigliceridemia severa. 2010;19:15-8

9. Miller M, Stone NJ, Ballantyne C, Bittner V, Criqui MH, Ginsberg HN, et al. Triglycerides and cardiovascular disease: A scientific statement from the American Heart Association. Circulation. 2011;123(20):2292-333.

10. Piepoli MF, Hoes AW, Agewall S, Albus C, Brotons C, Catapano AL, et al. 2016 European Guidelines on cardiovascular disease prevention in clinical practice. Eur Heart J. 2016;37(29):2315-81. 